\title{
Analysis of Methods for Multivariable Fre- quency Response Function Estimation in Closed Loop
}

Erik Wernholt, Svante Gunnarsson

Division of Automatic Control

E-mail: erikw@isy.liu.se, svante@isy.liu.se

9th March 2007

Report no.: LiTH-ISY-R-2775

Submitted to CDC 2007

Address:

Department of Electrical Engineering

Linköpings universitet

SE-581 83 Linköping, Sweden

WWW: http://WwW. control.isy.liu.se

AUTOMATIC CONTROL

REGLERTEKNIK

LINKÖPINGS UNIVERSITET

Technical reports from the Automatic Control group in Linköping are available from http://www. control.isy.liu.se/publications. 


\begin{abstract}
Estimation methods for the multivariable frequency response function are analyzed, both in open and closed loop. Approximate bias and covariance expressions are derived for the closed loop case. The usefulness of these expressions is illustrated in simulations of an industrial robot where three different estimators are compared. The choice of estimator depends on the signal-to-noise ratio as well as the measurement setup and a bias-variance trade-off.
\end{abstract}

Keywords: Nonparametric identification, Multivariable systems, Closed Loop 


\title{
Analysis of Methods for Multivariable Frequency Response Function Estimation in Closed Loop
}

\author{
Erik Wernholt and Svante Gunnarsson
}

\begin{abstract}
Estimation methods for the multivariable frequency response function are analyzed, both in open and closed loop. Approximate bias and covariance expressions are derived for the closed loop case. The usefulness of these expressions is illustrated in simulations of an industrial robot where three different estimators are compared. The choice of estimator depends on the signal-to-noise ratio as well as the measurement setup and a bias-variance trade-off.
\end{abstract}

\section{INTRODUCTION}

This paper mainly studies the properties of a frequency response function (FRF) estimation method for multivariable systems, described in [1], for both open loop and closed loop data. It is known, see for example [2], that this type of frequency domain method will give biased estimates when using closed loop data, but there are practical reasons why it anyway is motivated to study this problem. First, in many applications it is more or less inevitable to use feedback control during the data collection, and, second, the frequency domain identification method has appealing properties in its simplicity.

Previous studies of the performance of the method are presented in, e.g., [1] and [3] for open loop data and [4] contains some early work for closed loop data. In this paper, the FRF estimation method will be analyzed for both open loop and closed loop data. The open loop covariance expressions from the two papers [1] and [3] will here be combined. For closed loop data, an asymptotic expression for the bias is derived together with approximate covariance expressions. For numerical illustration, a simulation model of an industrial robot will be used. The robot application is interesting since it gives many challenging problems for system identification methods, such as a multivariable nonlinear system, oscillatory behavior, and data collection under feedback control. An overview of identification in robotics can be found in [5]. See also [6], [7], [8] for examples where FRF estimates are used for the identification of parametric robot models. The simulation results are evaluated using the derived bias and variance expressions. Corresponding results using experimental data from real robots are presented in, e.g., [9] and [10].

\section{The IDENTIFICATION METHOD}

The setup considered in this paper is given by

$$
y(t)=G(q)\left(u(t)+v_{u}(t)\right)+v_{y}(t),
$$

This work was supported by the VINNOVA competence center ISIS at Linköping University and the Swedish Research Council (VR).

E. Wernholt and S. Gunnarsson are with the Department of Electrical Engineering, Linköpings universitet, SE-58183 Linköping, Sweden \{erikw, svante\}@isy.liu.se where $G(q)$ is the $n_{y} \times n_{u}$ multivariable discrete-time transfer operator, with $q$ being the shift operator, and $v_{u}(t)$ and $v_{y}(t)$ are noise on the input and output, respectively. The input and output signals, $u(t) \in \mathbb{R}^{n_{u}}$ and $y(t) \in \mathbb{R}^{n_{y}}$, are measured at time instants $t_{n}=n T_{s}, n=1,2, \ldots, N$, with sample time $T_{s}$. Equation (1) can actually be transformed to an output noise case,

$$
y(t)=G(q) u(t)+v(t), \quad v(t)=G(q) v_{u}(t)+v_{y}(t) .
$$

To avoid leakage effects in the discrete Fourier transform (DFT), which is used by the identification method, the input signal, $u(t)$, is assumed to be $N_{P}$-periodic and an integer number of periods, $P$, of the steady state response is collected, giving $N=P N_{p}$ samples for each experiment. Consider now the DFTs of the measured input and output signals

$$
\begin{aligned}
& U\left(\omega_{k}\right)=\frac{1}{\sqrt{N}} \sum_{n=1}^{N} u\left(n T_{s}\right) e^{j \omega_{k} n T_{s}}, \\
& Y\left(\omega_{k}\right)=\frac{1}{\sqrt{N}} \sum_{n=1}^{N} y\left(n T_{s}\right) e^{j \omega_{k} n T_{s}},
\end{aligned}
$$

where only the $N_{P}$ frequencies $\omega_{k}=k_{\frac{2 \pi}{N_{P} T_{s}}}, \quad k=$ $1,2, \ldots, N_{P}$ are considered. Given the periodic data, the following linear mapping will hold exactly in the noise free case

$$
Y\left(\omega_{k}\right)=G\left(e^{j \omega_{k} T_{s}}\right) U\left(\omega_{k}\right),
$$

where $G\left(e^{j \omega_{k} T_{s}}\right) \in \mathbb{C}^{n_{y} \times n_{u}}$ is the FRF. To be able to extract $G\left(e^{j \omega_{k} T_{s}}\right)$ from data, at least $n_{u}$ different experiments are needed. The data vectors from $n_{e} \geq n_{u}$ different experiments will be collected into matrices (bold face in the sequel) where each column corresponds to one experiment. The inputoutput relation can then be written as

$$
\mathbf{Y}\left(\omega_{k}\right)=G\left(e^{j \omega_{k} T_{s}}\right) \mathbf{U}\left(\omega_{k}\right),
$$

where $\mathbf{U}\left(\omega_{k}\right) \in \mathbb{C}^{n_{u} \times n_{e}}$ and $\mathbf{Y}\left(\omega_{k}\right) \in \mathbb{C}^{n_{y} \times n_{e}}$. If $\mathbf{U}\left(\omega_{k}\right)$ has rank $n_{u}$, an estimate of $G\left(e^{j \omega_{k} T_{s}}\right)$ can be formed using the $H_{1}$ estimator (see also (10))

$$
\hat{G}\left(e^{j \omega_{k} T_{s}}\right)=\mathbf{Y}\left(\omega_{k}\right) \mathbf{U}^{\dagger}\left(\omega_{k}\right),
$$

where $\mathbf{U}^{\dagger}\left(\omega_{k}\right)$ is the pseudo-inverse $\mathbf{U}^{\dagger}=\mathbf{U}^{H}\left(\mathbf{U U}^{H}\right)^{-1}$ and $(\cdot)^{H}$ denotes complex conjugate transpose. When the measurements are corrupted by noise, (4) changes to

$$
\begin{aligned}
\mathbf{Y}\left(\omega_{k}\right) & =G\left(e^{j \omega_{k} T_{s}}\right) \mathbf{U}\left(\omega_{k}\right)+\mathbf{V}\left(\omega_{k}\right), \\
\mathbf{V}\left(\omega_{k}\right) & =\mathbf{V}_{y}\left(\omega_{k}\right)+G\left(e^{j \omega_{k} T_{s}}\right) \mathbf{V}_{u}\left(\omega_{k}\right) .
\end{aligned}
$$


The $\mathrm{H}_{1}$ estimator (5) can still be used, but the estimate will contain errors due to the noise.

In general the choice of excitation signal offers a large freedom in terms of frequency contents, magnitude, etc, as long as the matrix $\mathbf{U}\left(\omega_{k}\right)$ has rank $n_{u}$. In this paper, the orthogonal random phase multisine signal will be used. This signal has been suggested in [3] and [11] to minimize the variance ( $\operatorname{det} \sigma_{\hat{G}}^{2}$, cf. (16)) given certain amplitude constraints for the input signal. Assuming $n_{e}=M \cdot n_{u}, \mathbf{U}\left(\omega_{k}\right)$ is partitioned in $M$ blocks of size $n_{u} \times n_{u}$ as

$$
\begin{aligned}
\mathbf{U}\left(\omega_{k}\right) & =\left[\begin{array}{lll}
\mathbf{U}^{(1)}\left(\omega_{k}\right) & \ldots & \mathbf{U}^{(M)}\left(\omega_{k}\right)
\end{array}\right], \\
\mathbf{U}^{(m)}\left(\omega_{k}\right) & =\mathbf{U}_{\text {diag }}^{(m)}\left(\omega_{k}\right) \mathbf{T}, \quad m=1, \ldots, M,
\end{aligned}
$$

with $\mathbf{U}_{\text {diag }}^{(m)}\left(\omega_{k}\right)=\operatorname{diag}\left\{U_{l}^{(m)}\left(\omega_{k}\right)\right\}_{l=1}^{n_{u}}$ a diagonal matrix where each $U_{l}^{(m)}\left(\omega_{k}\right)$ is a random phase multisine signal, and $\mathbf{T}$ is an orthogonal matrix. A scalar random phase multisine signal $u(t)$ can be written as

$$
u(t)=\sum_{k=1}^{n_{f}} A_{k} \sin \left(\omega_{k} t+\phi_{k}\right),
$$

with amplitudes $A_{k}$, frequencies $\omega_{k}$ chosen from the grid $\left\{\frac{2 \pi l}{N_{P} T_{s}}, l=1, \ldots, N_{P} / 2-1\right\}$, and random phases $\phi_{k}$ uniformly distributed in $[0,2 \pi)$. The optimal matrix $\mathbf{T}$, with amplitude constraints $\left|\mathbf{T}_{i l}\right| \leq 1$, is given by [11]

$$
\mathbf{T}_{i l}=e^{\frac{2 \pi j}{n_{u}}(i-1)(l-1)} .
$$

For the single input, single output (SISO) case, a number of different FRF estimators have been suggested in the literature (see, e.g., [12] and [13]), which all have different properties regarding bias (for the output noise case only in closed loop) and variance. These estimators can often be generalized to the multiple input, multiple output (MIMO) case. If, for example, (7a) is used, (5) can be rewritten as

$$
\hat{G}=\left[\frac{1}{M} \sum_{m=1}^{M} \mathbf{Y}^{(m)} \mathbf{U}^{(m) H}\right]\left[\frac{1}{M} \sum_{m=1}^{M} \mathbf{U}^{(m)} \mathbf{U}^{(m) H}\right]^{-1} .
$$

(For notational simplicity, the frequency argument will be omitted when not explicitly needed.) This is why (5) and (10) will be called the $H_{1}$ estimator (cf. [12]). Another useful estimator is the arithmetic mean estimator

$$
\hat{G}^{\mathrm{ARI}}=\frac{1}{M} \sum_{m=1}^{M} \hat{G}^{(m)}=\frac{1}{M} \sum_{m=1}^{M} \mathbf{Y}^{(m)}\left[\mathbf{U}^{(m)}\right]^{-1} .
$$

In case (7) with (9) is used as input with the same amplitude distribution over the $M$ blocks $\left(U_{l}^{(m)}=U_{l}, m=1, \ldots, M\right)$, the $\mathrm{H}_{1}$ and ARI estimators, (10) and (11), will give the same estimate. The $\mathrm{H}_{1}$ estimator is more robust than the ARI estimator if certain blocks have poor SNR, but will typically give a larger bias for moderate SNR's. If the reference signal is measured, an asymptotically $(M \rightarrow \infty)$ unbiased estimator has been proposed in [13], which can be generalized to the

\section{MIMO case as}

$$
\hat{G}^{\mathrm{JIO}}=\left[\frac{1}{M} \sum_{m=1}^{M} \mathbf{Y}^{(m)} \mathbf{R}^{(m) H}\right]\left[\frac{1}{M} \sum_{m=1}^{M} \mathbf{U}^{(m)} \mathbf{R}^{(m) H}\right]^{-1},
$$

where $\mathbf{R}$ is the reference DFT matrix (cf. (21)) and JIO stands for joint input-output estimator. If the same excitation is used in all blocks and the measurements are synchronized, then (12) reduces to the errors-in-variables estimator [12]

$$
\begin{aligned}
& \hat{G}^{\mathrm{EIV}}=\left[\frac{1}{M} \sum_{m=1}^{M} \mathbf{Y}^{(m)}\right]\left[\frac{1}{M} \sum_{m=1}^{M} \mathbf{U}^{(m)}\right]^{-1} . \\
& \text { III. OPEN LOOP ERROR ANALYSIS }
\end{aligned}
$$

\section{OPEN LOOP ERROR ANALYSIS}

In this section, the $\mathrm{H}_{1}$ estimator (5) will be analyzed for open loop data. This analysis is closely related to the work in [1] and [3]. In [1] the analysis is conducted within an errorsin-variables ${ }^{1}$ framework, but the same covariance expression is obtained as for the the output noise case in this paper (see Theorem 1). In [3], covariance expressions are derived when the orthogonal multisine signal (7) is used as input. The MIMO system is there viewed as $n_{y}$ separate MISO systems with output noise. Implicitly, the output noise is assumed to be independent over the different outputs so expressions for the covariance between different rows in $\hat{G}$ are not presented. In this paper, the MIMO system will be considered without such noise assumption, which can be seen as applying (7) to Theorem 1. In addition, in Section IV the results will be extended to the closed loop case.

\section{A. Noise Assumptions}

For the analysis, we will assume the following noise properties.

Assumption 1: For each of the $n_{e}$ experiments, the DFTs of the input and output noise, $V_{u}\left(\omega_{k}\right)$ and $V_{y}\left(\omega_{k}\right)$ satisfy

$$
\begin{gathered}
\mathrm{E} V_{y}\left(\omega_{k}\right)=0, \quad \mathrm{E} V_{u}\left(\omega_{k}\right)=0, \\
\mathrm{E} V_{y}\left(\omega_{k}\right) V_{y}^{T}\left(\omega_{k}\right)=0, \quad \operatorname{E} V_{y}\left(\omega_{k}\right) V_{y}^{H}\left(\omega_{k}\right)=\sigma_{V_{y}}^{2}\left(\omega_{k}\right), \\
\mathrm{E} V_{u}\left(\omega_{k}\right) V_{u}^{T}\left(\omega_{k}\right)=0, \quad \operatorname{E~} V_{u}\left(\omega_{k}\right) V_{u}^{H}\left(\omega_{k}\right)=\sigma_{V_{u}}^{2}\left(\omega_{k}\right), \\
\operatorname{E~} V_{u}\left(\omega_{k}\right) V_{y}^{T}\left(\omega_{k}\right)=\operatorname{E} V_{u}\left(\omega_{k}\right) V_{y}^{H}\left(\omega_{k}\right)=0,
\end{gathered}
$$

for $k=1, \ldots, n_{f}$, which means that $V_{u}\left(\omega_{k}\right)$ and $V_{y}\left(\omega_{k}\right)$ are independent and circular complex. In addition, it is assumed that they are independent and identically distributed over the $n_{e}$ different experiments.

This assumption is common and justifiable in most practical circumstances ${ }^{2}$ and enables us to obtain the following result for the covariance of $\mathbf{V}\left(\omega_{k}\right)$.

Lemma 1: Consider the DFT matrix $\mathbf{V}\left(\omega_{k}\right) \in \mathbb{C}^{n_{y} \times n_{e}}$ in (6). Under Assumption 1, the covariance $\sigma_{\mathbf{V}}^{2}\left(\omega_{k}\right)$ is given by

$$
\sigma_{\mathbf{V}}^{2}\left(\omega_{k}\right)=\operatorname{Evec} \mathbf{V}\left(\omega_{k}\right)\left[\operatorname{vec} \mathbf{V}\left(\omega_{k}\right)\right]^{H}=I \otimes \sigma_{V}^{2}\left(\omega_{k}\right),
$$

where $\otimes$ is the Kronecker product and

$$
\sigma_{V}^{2}\left(\omega_{k}\right)=\sigma_{V_{y}}^{2}\left(\omega_{k}\right)+G\left(e^{j \omega_{k} T_{s}}\right) \sigma_{V_{u}}^{2}\left(\omega_{k}\right) G^{H}\left(e^{j \omega_{k} T_{s}}\right) .
$$

\footnotetext{
${ }^{1}$ Measurements $y(t)$ and $u(t)+v_{u}(t)$ with $y(t)=G(q) u(t)+v_{y}(t)$.

${ }^{2}$ For example, the DFT of filtered white noise is asymptotically circular complex when the number of samples, $N \rightarrow \infty$, see [14, Theorem 14.25].
} 
Proof: Let $\mathbf{V}_{i}$ denote column $i$ in $\mathbf{V} . \sigma_{\mathbf{V}}^{2}$ will then be a block matrix with blocks $\mathrm{E} \mathbf{V}_{i} \mathbf{V}_{j}^{H} \in \mathbb{C}^{n_{y} \times n_{y}}$. Expanding this expression gives

$$
\begin{aligned}
\mathrm{E} \mathbf{V}_{i} \mathbf{V}_{j}^{H}= & \mathrm{E} \mathbf{V}_{y, i} \mathbf{V}_{y, j}^{H}+\mathrm{E} \mathbf{V}_{y, i} \mathbf{V}_{u, j}^{H} G^{H}+ \\
& +\mathrm{E} G \mathbf{V}_{u, i} \mathbf{V}_{y, j}^{H}+\mathrm{E} G \mathbf{V}_{u, i} \mathbf{V}_{u, j}^{H} G^{H} \\
= & \begin{cases}\sigma_{V_{y}}^{2}+G \sigma_{V_{u}}^{2} G^{H} & i=j \\
0 & i \neq j\end{cases}
\end{aligned}
$$

where the last step follows from Assumption 1. $\sigma_{\mathbf{V}}^{2}$ will therefore be a block-diagonal matrix with $\sigma_{V}^{2}$ in all the diagonal blocks, which can be written as $I \otimes \sigma_{V}^{2}$.

\section{B. Bias and Covariance}

The bias and variance of the $\mathrm{H}_{1}$ estimator (5) will now be derived in the following theorem. The same result (for the case $n_{e}=n_{u}$ ) is stated without proof in [1] as a generalization of [15, Theorem 8.2.5] to the errors-in-variables framework. Here, a proof will be given for the output noise case, see also [15, Theorem 8.2.5].

Theorem 1: Consider the $\mathrm{H}_{1}$ estimator (5) and assume an open loop setup (6) where $\mathbf{U}\left(\omega_{k}\right)$ and $\mathbf{V}\left(\omega_{k}\right)$ are independent and the noise fulfills Assumption 1. Then, the estimate $\hat{G}\left(e^{j \omega_{k} T_{s}}\right)$ will be unbiased and the covariance $\sigma_{\hat{G}}^{2}\left(\omega_{k}\right)$ is given by

$$
\begin{aligned}
& \sigma_{\hat{G}}^{2}\left(\omega_{k}\right)=\operatorname{Evec} \tilde{G}\left(e^{j \omega_{k} T_{s}}\right)\left[\operatorname{vec} \tilde{G}\left(e^{j \omega_{k} T_{s}}\right)\right]^{H}= \\
& =\left[\mathbf{U}\left(\omega_{k}\right) \mathbf{U}^{H}\left(\omega_{k}\right)\right]^{-T} \otimes \sigma_{V}^{2}\left(\omega_{k}\right),
\end{aligned}
$$

with $\tilde{G}=\hat{G}-G$ and $\sigma_{V}^{2}$ from (15).

Proof: The estimation error $\tilde{G}$ can be rewritten as

$$
\tilde{G}=\hat{G}-G=(G \mathbf{U}+\mathbf{V}) \mathbf{U}^{\dagger}-G=\mathbf{V U}^{\dagger},
$$

and $\mathrm{E} \tilde{G}=0$ follows since $\mathrm{E} \mathbf{V}=0$ and $\mathbf{U}$ and $\mathbf{V}$ are independent. For the covariance, vec $\tilde{G}$ can be rewritten as ${ }^{3}$ $\operatorname{vec} \tilde{G}=\left(\mathbf{U}^{\dagger T} \otimes I\right) \operatorname{vec} \mathbf{V}$ by using $\operatorname{vec}(A D B)=\left(B^{T} \otimes\right.$ $A$ ) vec $D$. Equation (16) then follows from

$$
\begin{aligned}
\sigma_{\hat{G}}^{2} & =\mathrm{E} \operatorname{vec} \tilde{G}[\operatorname{vec} \tilde{G}]^{H} \\
& =\mathrm{E}\left[\mathbf{U}^{\dagger T} \otimes I\right] \operatorname{vec} \mathbf{V}(\operatorname{vec} \mathbf{V})^{H}\left[\mathbf{U}^{\dagger T} \otimes I\right]^{H} \\
& \left.=\left[\mathbf{U}^{\dagger T} \otimes I\right]\left[I \otimes \sigma_{V}^{2}\right] \overline{\mathbf{U}^{\dagger}} \otimes I\right] \\
& =\left[\mathbf{U}^{\dagger T} \overline{\mathbf{U}^{\dagger}}\right] \otimes \sigma_{V}^{2}=\left[\mathbf{U}^{\dagger H} \mathbf{U}^{\dagger}\right]^{T} \otimes \sigma_{V}^{2} \\
& =\left[\left(\mathbf{U} \mathbf{U}^{H}\right)^{-1} \mathbf{U} \mathbf{U}^{H}\left(\mathbf{U} \mathbf{U}^{H}\right)^{-1}\right]^{T} \otimes \sigma_{V}^{2},
\end{aligned}
$$

where $(A \otimes B)(C \otimes D)=(A B) \otimes(C D)$ has been used twice in the third step.

Continuing the error analysis with the orthogonal multisine signal (7) gives the following results (cf. [3]).

Corollary 1: Using an orthogonal multisine signal (7) with $\mathbf{T}$ from (9) simplifies $\sigma_{\hat{G}}^{2}$ in Theorem 1 to

$$
\sigma_{\hat{G}}^{2}=\operatorname{diag}\left\{\frac{1}{n_{u} \sum_{m=1}^{M}\left|U_{l}^{(m)}\right|^{2}}\right\}_{l=1}^{n_{u}} \otimes \sigma_{V}^{2},
$$

\footnotetext{
${ }^{3}$ Using basic properties for the Kronecker product of complex matrices,
} see for example [14, p. 420]. and the variance of each element in $\hat{G}$ as

$$
\sigma_{\hat{G}_{i j}}^{2}=\frac{\sigma_{V, i i}^{2}}{n_{u} \sum_{m=1}^{M}\left|U_{j}^{(m)}\right|^{2}} .
$$

Proof: Follows by noting that $\mathbf{T T}^{H}=n_{u} I$ for (9) as

$$
\begin{aligned}
\mathbf{U U}^{H} & =\sum_{m} \mathbf{U}^{(m)} \mathbf{U}^{(m) H}=\sum_{m} \mathbf{U}_{\text {diag }}^{(m)} \mathbf{T} \mathbf{T}^{H} \mathbf{U}_{\text {diag }}^{(m) H} \\
& =n_{u} \sum_{m} \mathbf{U}_{\text {diag }}^{(m)} \mathbf{U}_{\text {diag }}^{(m) H}=n_{u} \sum_{m} \operatorname{diag}\left\{\left|U_{l}^{(m)}\right|^{2}\right\}_{l=1}^{n_{u}} .
\end{aligned}
$$

This means that different columns in $\hat{G}$ are uncorrelated and that the covariance for a certain column is inversely proportional to the total input power in that particular input channel. Note that if another type of excitation signal $\mathbf{U}$ is used, without the property that $\mathbf{U} \mathbf{U}^{H}$ is diagonal, then the columns in $\hat{G}$ will be correlated. The variance (19) is given by a noise-to-signal ratio, where $\sigma_{V i i}^{2}$ is the noise variance in output $i$ and the denominator is the total power for input $j$ during the $n_{e}$ experiments.

\section{Closed Loop ERror Analysis}

In this paper the aim is to also analyze the properties of the identification method in a closed loop setup . Therefore, let the input be given by

$$
u(t)=F(q)(r(t-y(t))
$$

where $F(q)$ is the controller and $r(t) \in \mathbb{R}^{n_{y}}$ is the reference signal. For simplicity, $n_{y}=n_{u}$ will be assumed in this section ( $n_{y} \geq n_{u}$ is enough for most expressions). The input DFT matrix $\mathbf{U}\left(\omega_{k}\right)$ is then given by

$$
\mathbf{U}\left(\omega_{k}\right)=G_{u}\left(e^{j \omega_{k} T_{s}}\right)\left(\mathbf{R}\left(\omega_{k}\right)-\mathbf{V}\left(\omega_{k}\right)\right),
$$

where

$$
G_{u}(q)=(I+F(q) G(q))^{-1} F(q)
$$

\section{A. Bias}

Since $\mathbf{U}$ and $\mathbf{V}$ now are correlated, the FRF estimate $\hat{G}$ in (5) using the $\mathrm{H}_{1}$ estimator will be biased. To analyze the bias, we will assume a closed loop setup according to (2) and (20), where the noise fulfills Assumption 1 and $\mathbf{R}$ and $\mathrm{V}$ are independent. The FRF estimation error is given by

$$
\tilde{G}=\hat{G}-G=\mathbf{V} \mathbf{U}^{\dagger}=\mathbf{V}(\mathbf{R}-\mathbf{V})^{\dagger} G_{u}^{-1}
$$

Calculating the bias $\mathrm{E} \tilde{G}$ seems hard due to the matrix inverse. Expanding $\mathbf{V}(\mathbf{R}-\mathbf{V})^{\dagger}$ gives

$$
\begin{aligned}
\tilde{G}= & {\left[\frac{1}{n_{e}} \sum_{i=1}^{n_{e}} \mathbf{V}_{i} \mathbf{R}_{i}^{H}-\frac{1}{n_{e}} \sum_{i=1}^{n_{e}} \mathbf{V}_{i} \mathbf{V}_{i}^{H}\right] \times } \\
& {\left[\frac{1}{n_{e}} \sum_{i=1}^{n_{e}} \mathbf{R}_{i} \mathbf{R}_{i}^{H}+\frac{1}{n_{e}} \sum_{i=1}^{n_{e}} \mathbf{V}_{i} \mathbf{V}_{i}^{H}\right.} \\
& \left.-\frac{1}{n_{e}} \sum_{i=1}^{n_{e}} \mathbf{R}_{i} \mathbf{V}_{i}^{H}-\frac{1}{n_{e}} \sum_{i=1}^{n_{e}} \mathbf{V}_{i} \mathbf{R}_{i}^{H}\right]^{-1} G_{u}^{-1}
\end{aligned}
$$

These sums of independent (over $i$ ) variables can, by the law of large numbers, be replaced by expectation as the number 
of experiments, $n_{e}$, tends to infinity. Assuming that $\mathbf{R}$ and $\mathbf{V}$ are independent then gives

$$
\lim _{n_{e} \rightarrow \infty} \tilde{G}=-\sigma_{V}^{2}\left[\sigma_{R}^{2}+\sigma_{V}^{2}\right]^{-1} G_{u}^{-1}
$$

where $\sigma_{R}^{2}=\lim _{n_{e} \rightarrow \infty} \frac{1}{n_{e}} \sum_{i=1}^{n_{e}} \mathbf{R}_{i} \mathbf{R}_{i}^{H}$. Even though this asymptotic error expression is not technically the bias $\mathrm{E} \tilde{G}$, it still gives valuable information about the bias one could expect when $M$ is reasonably large. Using $G_{u}^{-1}=G+F^{-1}$ finally gives

$$
\lim _{n_{e} \rightarrow \infty} \hat{G}=\sigma_{R}^{2}\left[\sigma_{R}^{2}+\sigma_{V}^{2}\right]^{-1} G-\sigma_{V}^{2}\left[\sigma_{R}^{2}+\sigma_{V}^{2}\right]^{-1} F^{-1} .
$$

For frequencies where the SNR is poor, the estimate will tend to the inverse controller. If $\sigma_{V}^{2}$ and $\sigma_{R}^{2}$ are diagonal, (24) simplifies to

$$
\lim _{n_{e} \rightarrow \infty} \tilde{G}_{i j}=-\frac{\sigma_{V, i i}^{2}}{\sigma_{V, i i}^{2}+\sigma_{R, i i}^{2}}\left(G_{i j}+F_{i j}^{-1}\right),
$$

such that the relative error $\left(\tilde{G}_{i j} / G_{i j}\right)$ will be (approximately) proportional to the noise-to-signal ratio.

These bias expressions could be compared with the bias for the ARI estimator (11) in the SISO case [12]

$$
\mathrm{E} \tilde{G}^{\mathrm{ARI}}=-e^{-\sigma_{R}^{2} / \sigma_{V}^{2}} G_{u}^{-1}
$$

and

$$
\mathrm{E} \hat{G}^{\mathrm{ARI}}=\left(1-e^{-\sigma_{R}^{2} / \sigma_{V}^{2}}\right) G-e^{-\sigma_{R}^{2} / \sigma_{V}^{2}} F^{-1},
$$

which indicates a huge difference for large SNR's. A similar difference can be seen also in the MIMO case. When the SNR is small, on the other hand, the ARI estimator will deteriorate since $\mathbf{U}^{(m)}$ could loose rank for some blocks, giving infinite covariance. The covariance $\sigma_{\hat{G}}^{2}$ for the ARI estimator (11) can easily be derived, similarly to the proof of Theorem 1,

$$
\sigma_{\hat{G}}^{2}=\frac{1}{M^{2}} \sum_{m=1}^{M}\left[\mathbf{U}^{(m)} \mathbf{U}^{(m) H}\right]^{-T} \otimes \sigma_{V}^{2},
$$

which equals the covariance of the $\mathrm{H}_{1}$ estimator (5) (cf. (16)) in case all $\mathbf{U}^{(m)}$ have the same amplitude. In other cases, the variance is typically larger for the ARI estimator. If the reference signal is measured, then the JIO estimator (12) is a good candidate since it is asymptotically $(M \rightarrow \infty)$ unbiased and have approximately the same variance as the $\mathrm{H}_{1}$ estimator. The selection of estimator is therefore, as usual, a bias-variance trade-off.

A problem with the asymptotic bias expressions (24) (26) is that for large SNR's, $n_{e}$ must be fairly large until these expressions are useful, mainly due to the variance of $X=\frac{1}{n_{e}} \sum_{i=1}^{n_{e}} \mathbf{V}_{i}\left(\mathbf{R}_{i}-\mathbf{V}_{i}\right)^{H}$. Assume, for simplicity, that $\sigma_{V}^{2}$ and $\sigma_{R}^{2}$ are diagonal. The matrix $X$, with mean $-\sigma_{V}^{2}$, then has variance

$$
\mathrm{E} X_{i j} X_{i j}^{H}=\frac{1}{n_{e}}\left(\sigma_{V, i i}^{2} \sigma_{R, j j}^{2}+\sigma_{V, i i}^{2} \sigma_{V, j j}^{2}\right) .
$$

For this matrix to be diagonal dominant, we require

$$
n_{e} \gg\left(\sigma_{R, j j}^{2}+\sigma_{V, j j}^{2}\right) / \sigma_{V, i i}^{2}
$$

\section{B. Covariance}

For the remaining section, the bias will be neglected, using the approximation

$$
\tilde{G} \approx \mathbf{V R}^{\dagger} G_{u}^{-1}
$$

which can be viewed as neglecting the noise in the feedback loop as

$$
\mathbf{U} \approx G_{u} \mathbf{R}
$$

Inserting (33) into Theorem 1 yields:

Theorem 2: Consider the $\mathrm{H}_{1}$ estimator (5) and assume a closed loop setup according to (2) and (20), where the noise fulfills Assumption 1. For large SNR $(\mathbf{V} \ll \mathbf{R})$ the covariance $\sigma_{\hat{G}}^{2}\left(\omega_{k}\right)$ is approximately given by

$$
\sigma_{\hat{G}}^{2}=\left[G_{u}^{-H}\left(\mathbf{R R}^{H}\right)^{-1} G_{u}^{-1}\right]^{T} \otimes \sigma_{V}^{2},
$$

with $G_{u}^{-1}=G+F^{-1}$.

Even though this covariance expression is approximate, it still gives valuable insight to how the covariance is affected by the closed loop setup. The validity of Theorem 2 will depend on the SNR. Now, similar expressions to Corollary 1 will be derived using the orthogonal multisine signal

$$
\begin{aligned}
\mathbf{R}\left(\omega_{k}\right) & =\left[\begin{array}{lll}
\mathbf{R}^{(1)}\left(\omega_{k}\right) & \ldots & \mathbf{R}^{(M)}\left(\omega_{k}\right)
\end{array}\right], \\
\mathbf{R}^{(m)}\left(\omega_{k}\right) & =\mathbf{R}_{\text {diag }}^{(m)}\left(\omega_{k}\right) \mathbf{T}, \quad m=1, \ldots, M,
\end{aligned}
$$

as in (7) with $\mathbf{T}$ from (9). This parameterization corresponds to an optimal experiment design given output amplitude constraints, compared to the open loop case (7) when having input amplitude constraints. If we actually have input constraints in the closed loop case, $\mathbf{R}=G_{u}^{-1} \mathbf{U}$ can be used, yielding $\mathbf{R}_{\text {diag }}=G_{u}^{-1} \mathbf{U}_{\text {diag. }}$. Note that $\mathbf{R}_{\text {diag }}$ then no longer is diagonal.

Corollary 2: Using an orthogonal multisine signal (35) with $\mathbf{T}$ from (9) simplifies $\sigma_{\hat{G}}^{2}$ in Theorem 2 to

$$
\sigma_{\hat{G}}^{2}=\left[G_{u}^{-H} \operatorname{diag}\left\{\frac{1}{n_{u} \sum_{m=1}^{M}\left|R_{l}^{(m)}\right|^{2}}\right\}_{l=1}^{n_{u}} G_{u}^{-1}\right]^{T} \otimes \sigma_{V}^{2},
$$

with $G_{u}^{-1}=G+F^{-1}$. The variance of each element in $\hat{G}$ can be written as

$$
\sigma_{\hat{G}_{i j}}^{2}=\sigma_{V, i i}^{2} \sum_{l=1}^{n_{u}} \frac{\left|\left(G+F^{-1}\right)_{l j}\right|^{2}}{n_{u} \sum_{m=1}^{M}\left|R_{l}^{(m)}\right|^{2}} .
$$

One immediately notes that, to reduce the variance, the reference signal should be as large as possible. In addition, a large gain controller will also reduce the variance. Actually, $F=-G^{-1}$, would give zero error but is unrealistic since it corresponds to a marginally stable system and infinite input power. If the noise variance is the same for the different outputs, then all elements in a column will have the same variance. The variance will typically vary between different columns so for a symmetric system $\left(\left|G_{i j}\right|=\left|G_{j i}\right|\right)$, the variance will usually be non-symmetric $\left(\sigma_{\hat{G}_{i j}}^{2} \neq \sigma_{\hat{G}_{j i}}^{2}\right)$. Small elements in a column will also, in general, have a larger relative error $\left(\sigma_{\hat{G}_{i j}} /\left|G_{i j}\right|\right)$. 


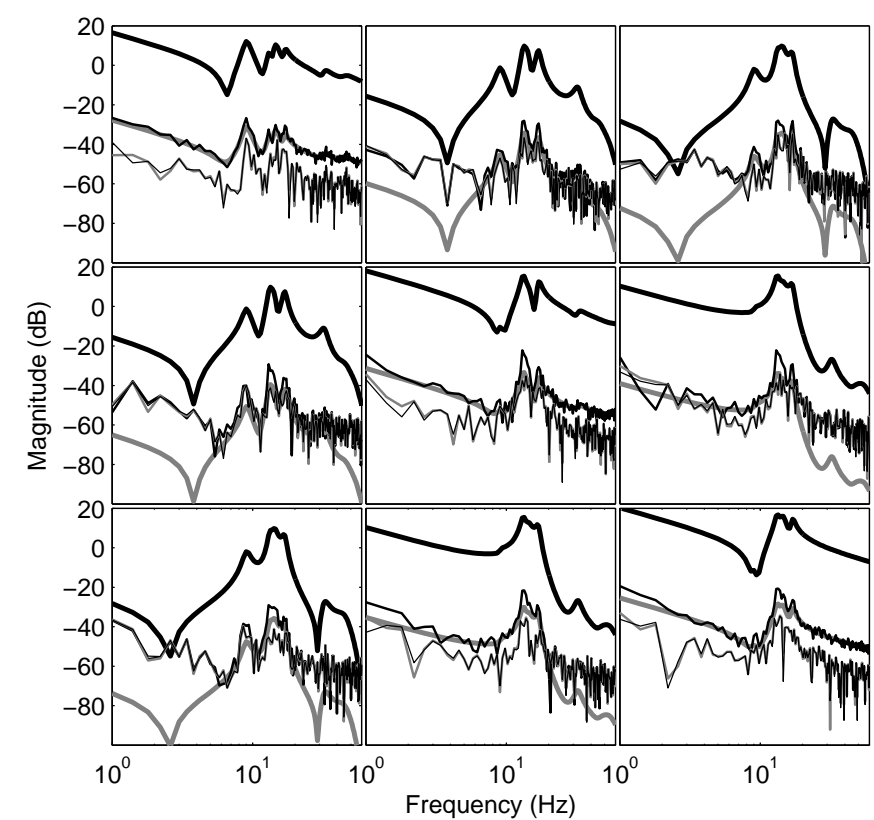

Fig. 1. Bias for the estimators with $22 \mathrm{~dB}$ SNR: $\mathrm{H}_{1}$ (black line), ARI (gray line), JIO (thin black line), together with the system $G$ (thick black line) and the bias expression (24) (thick gray line).

\section{NUMERICAL ILLUSTRATION}

As a numerical illustration, a simulation model of an industrial robot will be used. The model $G(q)$ is a linearized version of a nonlinear state-space model from [6] with 24 states, describing the dynamics of axes 1,2 and $3\left(n_{u}=\right.$ $n_{y}=3$ ) from applied motor torque to achieved motor velocity (the FRF of $G$ can be seen in all the figures). The controller $F(q)$ is a diagonal PI controller with a gain of $8 \mathrm{~dB}$ in the excited frequency interval. A sample period of $T_{s}=0.5 \mathrm{~ms}$ is used with $N=N_{P}=10^{4}$ samples from the steady state response.

As excitation, the orthogonal multisine signal (9) and (35) is used with a flat amplitude distribution from 2 to $60 \mathrm{~Hz}$ and $\mathbf{R}_{\text {diag }}^{(m)}$ the same in all $M=100$ blocks. For the noise $v$ in (2), $v_{u}=0$ and $v_{y}$ is normal distributed white noise, giving a diagonal covariance matrix $\sigma_{V}^{2}$. The resulting signalto-noise ratio, $\mathrm{SNR}_{i}=20 \log _{10}\left(\sigma_{R, i i} / \sigma_{V, i i}\right)$, is constant for the excited frequencies and simulations with two different SNR's, $3 \mathrm{~dB}$ and $22 \mathrm{~dB}$ will be presented. The statistical properties of the estimators are obtained by repeating the simulations 100 times with different noise realizations.

First, consider the $22 \mathrm{~dB}$ case and the three estimators: $\mathrm{H}_{1}$ (5), ARI (11) and JIO (12). The bias E $\tilde{G}$ and variance $\sigma_{\hat{G}}^{2}$ (actually the standard deviation $\sigma_{\hat{G}}$ ) of the estimators are plotted in Figs. 1 and 2, respectively. In addition are the expressions for bias (24) and variance (37) of the $\mathrm{H}_{1}$ estimator plotted. As can be seen in Fig. 2, the variance is approximately the same for the three estimators and the approximate variance expression is fairly accurate.

As was mentioned in the end of Section IV, the variance is non-symmetric, which can be seen by comparing elements $(1,3)$ and $(3,1)$ in Fig. 2. For small elements, the relative error

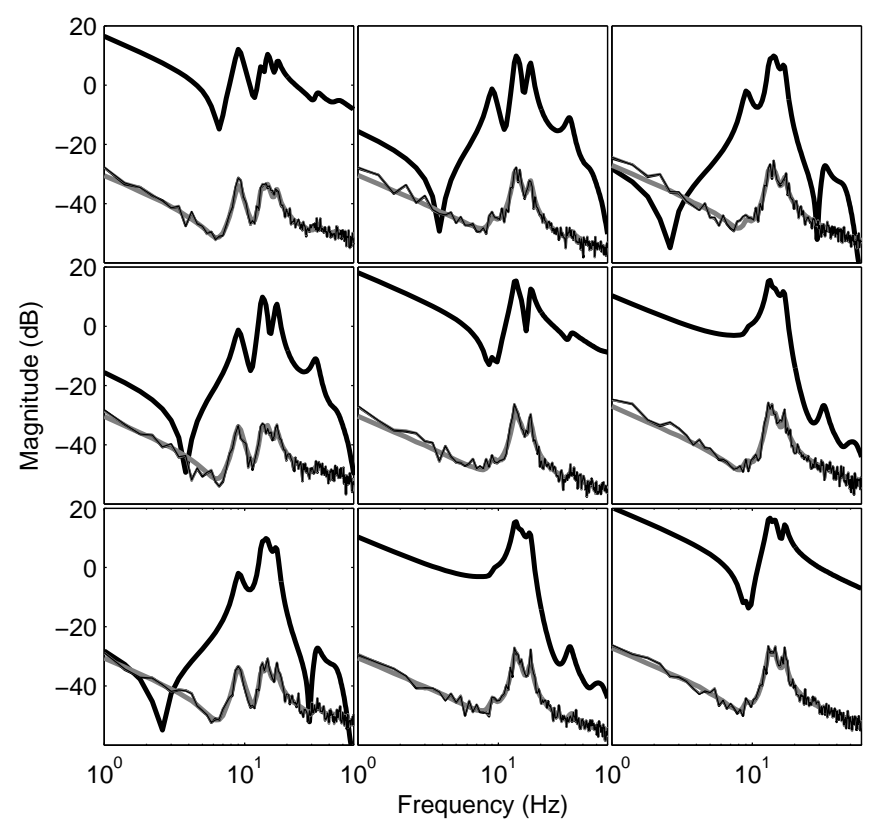

Fig. 2. Standard deviation for the estimators with $22 \mathrm{~dB}$ SNR: $\mathrm{H}_{1}$ (black line), ARI (gray line), JIO (thin black line), together with the system $G$ (thick black line) and the variance expression (37) (thick gray line).

is also larger, which can be seen by comparing elements $(1,1)$ and $(3,1)$ in Fig. 2 for low frequencies. This is inherent in all the studied estimation methods.

For the bias expression, see Fig. 1, the bias of the ARI and JIO estimators are approximately the same and always equal to or lower than for the $\mathrm{H}_{1}$ estimator. The asymptotic bias expression (24) is a lower limit for the bias of the $\mathrm{H}_{1}$ estimator and there is a good match when $G$ is large, especially for the diagonal elements. In this simulation, $\sigma_{V}^{2}$ and $\sigma_{R}^{2}$ are diagonal so the asymptotic expression (24) simplifies to (26). A problem is that with $M=100$, the off-diagonal elements of $\mathbf{V}(\mathbf{R}-\mathbf{V})^{\dagger}$ in (23) are still quite large. If all elements of $\mathbf{V}(\mathbf{R}-\mathbf{V})^{\dagger}$ would be equal to $\alpha$, then the bias is $\tilde{G}_{i j}=\alpha \sum_{l}\left(G_{l j}+F_{l j}^{-1}\right)$, i.e., the same bias for all elements in a given column. That is almost the case here since $M=100$ is not large enough, which explains the behavior for the off-diagonal elements in Fig. 1.

To see the different properties of the three estimators, the SNR is reduced to $3 \mathrm{~dB}$ and the bias and variance are plotted in Figs. 3 and 4, respectively. As can be seen, there is now a big difference both in bias and variance. The $\mathrm{H}_{1}$ estimator gives largest bias and smallest variance and the JIO estimator gives smallest bias and a smaller variance than the ARI estimator. The JIO estimator should therefore be used if the reference signal is measured. The variance expression (37) predicts the variance of the JIO estimator fairly accurately, but the bias expression (24) does not work here. The validity of the bias expression with respect to the SNR therefore needs further studies. 


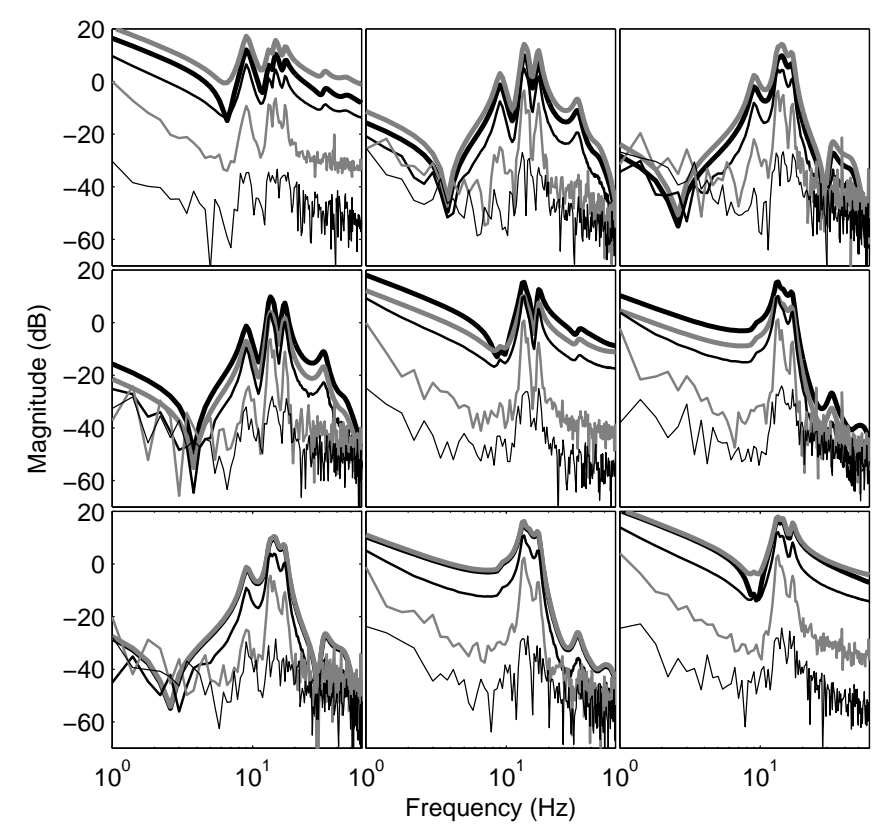

Fig. 3. Bias for the estimators with $3 \mathrm{~dB}$ SNR: $\mathrm{H}_{1}$ (black line), ARI (gray line), JIO (thin black line), together with the system $G$ (thick black line) and the bias expression (24) (thick gray line).

\section{Conclusions}

In this paper, FRF estimation methods for MIMO systems have been studied. The analysis has mainly focused on the $\mathrm{H}_{1}$ estimator, where expressions for the asymptotic bias and approximate covariance for closed loop data have been proposed. In addition have the arithmetic mean estimator (ARI) and the joint input-output estimator (JIO) been generalized to the MIMO case. The JIO estimator requires the reference signal to be known, but is asymptotically unbiased.

In the simulations, all three estimators give approximately the same variance for large SNR's and the bias of the $\mathrm{H}_{1}$ estimator is larger or equal to the bias of the ARI and JIO estimators. For small SNR's, the $\mathrm{H}_{1}$ estimator gives largest bias and smallest variance and the JIO estimator gives smallest bias and a smaller variance than the ARI estimator. Among these three estimators, the JIO estimator is the preferred choice if the reference signal is measured. Otherwise, the ARI estimator should be used if the SNR is guaranteed to be large enough in all blocks (since each $\mathbf{U}^{(m)}$ must be invertable, see also (29)). The $\mathrm{H}_{1}$ estimator is, in that respect, more robust than the ARI estimator.

The proposed variance expression (37) predicts the variance fairly accurately, but the bias expression (24) does not work for small SNR's.

\section{REFERENCES}

[1] P. Guillaume, R. Pintelon, and J. Schoukens, "Accurate estimation of multivariable frequency response functions," in Proceedings of the 13th IFAC Triennial World Congress, San Francisco, 1996, pp. 423428.

[2] L. Ljung, System Identification: Theory for the User, 2nd ed. Upper Saddle River, New Jersey, USA: Prentice Hall, 1999.

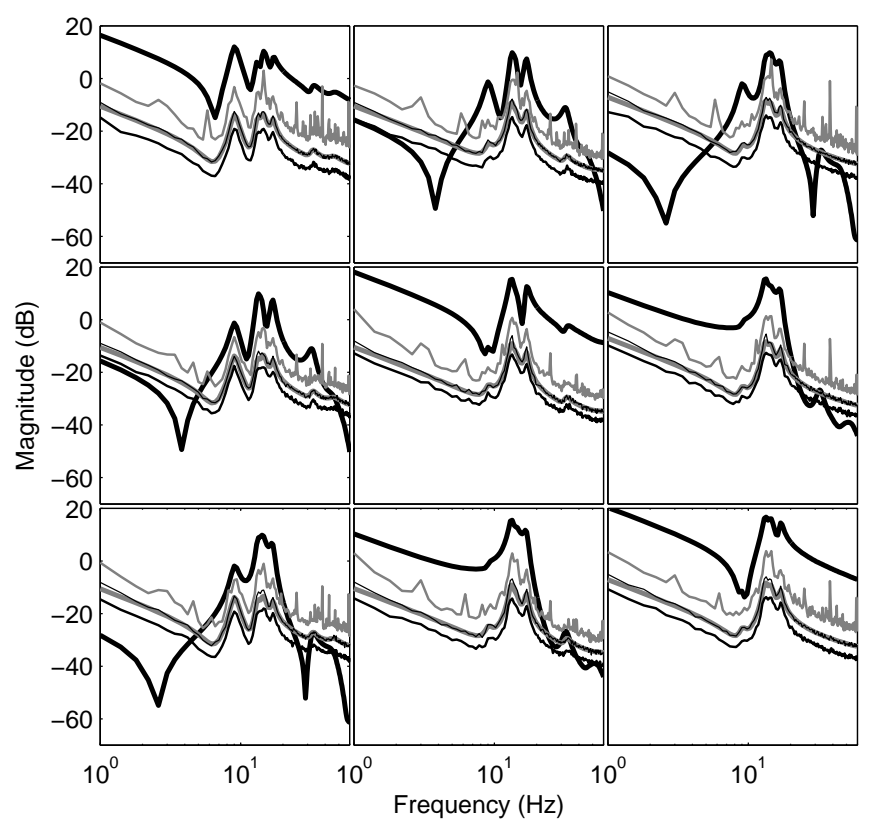

Fig. 4. Standard deviation for the estimators with $3 \mathrm{~dB}$ SNR: $\mathrm{H}_{1}$ (black line), ARI (gray line), JIO (thin black line), together with the system $G$ (thick black line) and the variance expression (37) (thick gray line).

[3] T. Dobrowiecki, J. Schoukens, and P. Guillaume, "Optimized excitation signals for MIMO frequency response function measurments systems." in Proc. 22nd IEEE Instrumentation and Measurement Technology Conference, 2005.

[4] E. Wernholt and S. Gunnarsson, "On the use of a multivariable frequency response estimation method for closed loop identification," in Proc. of the 43rd IEEE Conference on Decision and Control, Nassau, Bahamas, Dec 2004.

[5] K. Kozlowski, Modelling and identification in robotics, ser. Advances in Industrial Control. London: Springer, 1998.

[6] J. Öhr, S. Moberg, E. Wernholt, S. Hanssen, J. Pettersson, S. Persson, and S. Sander-Tavallaey, "Identification of flexibility parameters of 6-axis industrial manipulator models," in Proc. 2006 International Conference on Noise and Vibration Engineering (ISMA 2006), Leuven, Belgium, Sep 2006.

[7] E. Berglund and G. E. Hovland, "Automatic elasticity tuning of industrial robot manipulators," in Proceedings of the 39th IEEE Conference on Decision and Control, Sydney, Australia, Dec 2000, pp. 5091-5096.

[8] F. Khorrami, S. Jain, and A. Tzes, "Experimental results on adaptive nonlinear control and input preshaping for multi-link flexible manipulators," Automatica, vol. 31, no. 1, pp. 83-97, Jan 1995.

[9] E. Wernholt, "On multivariable and nonlinear identification of industrial robots," Department of Electrical Engineering, Linköping University, SE-581 83 Linköping, Sweden, Tech. Rep. Licentiate Thesis no. 1131, Dec 2004.

[10] E. Wernholt and S. Gunnarsson, "Detection and estimation of nonlinear distortions in industrial robots," in Proc. 23rd IEEE Instumentation and Measurement Technology Conference, Sorrento, Italy, Apr 2006, pp. 1913-1918.

[11] T. Dobrowiecki and J. Schoukens, "Measuring linear approximation to weakly nonlinear MIMO systems," in Proc. 16th IFAC World Congress, Prague, July 2005.

[12] R. Pintelon and J. Schoukens, "Measurement of frequency response functions using periodic excitations, corrupted by correlated input/output errors," IEEE Transactions on Instrumentation and Measurement, vol. 50, no. 6, pp. 1753-1760, Dec 2001.

[13] P. E. Wellstead, "Nonparametric methods of system identification," Automatica, vol. 17, no. 1, pp. 55-69, Jan 1981.

[14] R. Pintelon and J. Schoukens, System identification: a frequency domain approach. New York: IEEE Press, 2001.

[15] D. R. Brillinger, Time Series: Data Analysis and Theory, expanded ed. McGraw-Hill, New York, 1981. 


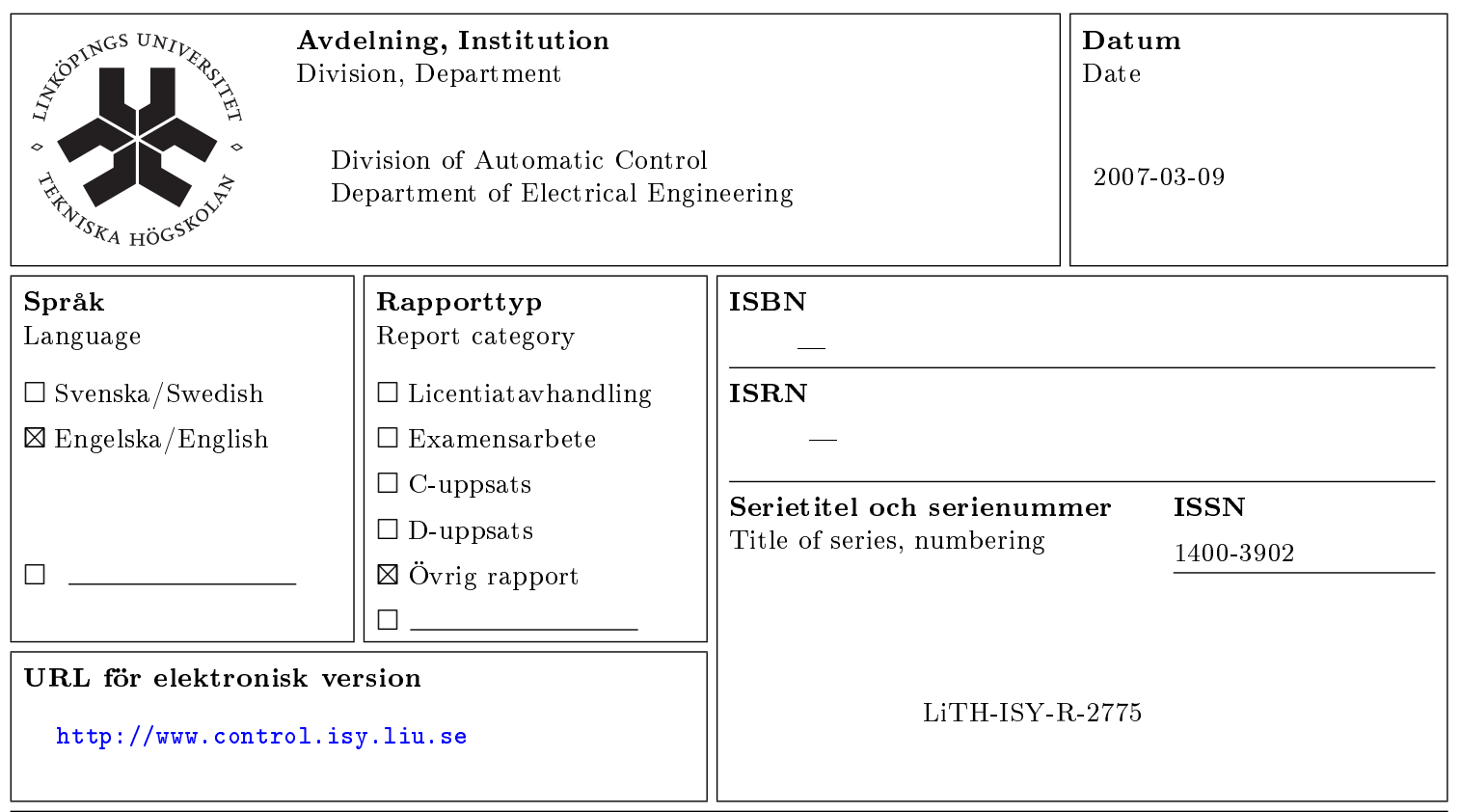

Titel Analysis of Methods for Multivariable Frequency Response Function Estimation in Closed Title Loop

Författare Erik Wernholt, Svante Gunnarsson

Author

\section{Sammanfattning}

Abstract

Estimation methods for the multivariable frequency response function are analyzed, both in open and closed loop. Approximate bias and covariance expressions are derived for the closed loop case. The usefulness of these expressions is illustrated in simulations of an industrial robot where three different estimators are compared. The choice of estimator depends on the signal-to-noise ratio as well as the measurement setup and a bias-variance trade-off. 